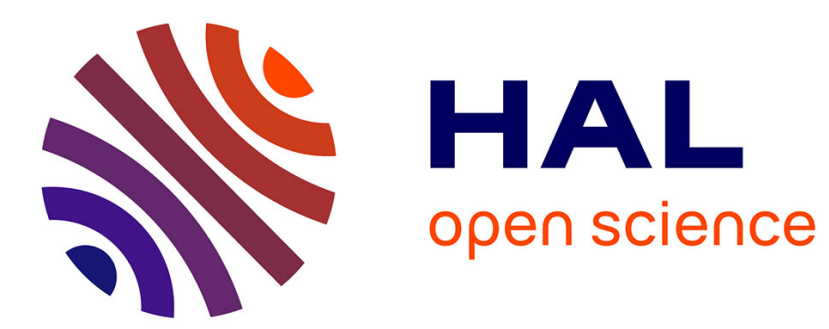

\title{
La centuriation romaine de la colonie de Valence
}

Albert Grenier

\section{To cite this version:}

Albert Grenier. La centuriation romaine de la colonie de Valence. Gallia - Fouilles et monuments archéologiques en France métropolitaine, 1958, 16 (2), pp.281-284. 10.3406/galia.1958.2236 . hal01924562

\section{HAL Id: hal-01924562 \\ https://hal.science/hal-01924562}

Submitted on 3 Mar 2020

HAL is a multi-disciplinary open access archive for the deposit and dissemination of scientific research documents, whether they are published or not. The documents may come from teaching and research institutions in France or abroad, or from public or private research centers.
L'archive ouverte pluridisciplinaire HAL, est destinée au dépôt et à la diffusion de documents scientifiques de niveau recherche, publiés ou non, émanant des établissements d'enseignement et de recherche français ou étrangers, des laboratoires publics ou privés.

\section{(ㅇ)(1) $\$$}

Distributed under a Creative Commons Attribution - NonCommercial - NoDerivatives| 4.0 


\title{
DOCUMENTS COMMENTÉS
}

\author{
La centuriation romaine de la colonie de Valence
}

Dans le très important volume que vient de publier M. John Bradford : Ancienl Landscapes (Londres, 1957), on trouve, pl. 50 (ad p. 206), la reproduction d'une photographie aérienne de la ville de Valence et, p. 207, fig. 20, un croquis résultant d'autres photographies qui représente les limites dont les traces subsistent, à l'Est de la ville, entre Valence et Chabeuil, l'ancienne Cerebelliaca mutatio de l'itinéraire de Bordeaux à Jérusalem, à XII milles = 18 kilomètres de Valence. Depuis la guerre où il avait fait son éducation, M. Bradford s'est affirmé, en de nombreux articles, comme le maître de l'application de la photographie aérienne à la recherche archéologique. Il en apporte, dans ce livre attachant, la théorie appuyée sur de nombreux exemples en même temps que bon nombre de renseignements pratiques. Il m'a semblé intéressant d'y relever ce qui concerne la Gaule romaine. Je remercie M. Bradford d'avoir bien voulu m'autoriser à reproduire le croquis qu'il donne de la limitatio du territoire de la colonie en direction de Ghabeuil ainsi que la belle photographie aérienne qu'il a publiée et à résumer pour les lecteurs de Gallia l'essentiel des constatations que lui a fournies l'examen de sa photographie aérienne.

De la photographie de la pl. 50 (fig. 2) on rapprochera le plan à plus grande échelle de la partie romaine de Valence qu'on trouvera (p. 87) dans le dernier fascicule de la FOR, Carle archéologique du département de la Drôme (fasc. XI, CNRS, 1957), dû au regrellé Chanoine Sautel et mis au point par M. P.-M. Duval. Ce plan a été établi avec le concours de M. André Blanc, de Valence. On y trouvera les rues modernes avec leurs noms. Il donne, pour ainsi dire, l'interprétation de la photographie aérienne. Le cardo, bien visible sur la photo, est marqué sur le plan par un trait pointillé qui coupe la ville à peu près en deux moitiés. Il continue la voie maîtresse de la vallée du Rhône, bien visible sur la photo à l'approche de la ville. La voie entrait dans Valence par la Porte du Sud connue par des fouilles; elle en sortait par la Porte Tourdéon, aujourd'hui disparue, mais dont on sait l'emplacement à l'intérieur de l'ancienne caserne. On reconnaît fort bien, sur le plan, le decumanus, visible sur la photo aérienne; il vient couper le cardo perpendiculairement, toujours vers le milieu de la ville, devant l'Hòtel de Ville, place de la Liberté entre l'Hôtel de Ville et le Théâtre. Là devait être le Forum, qui est resté le centre de la ville moderne. Ce decumanus central (rue Madier-Montjau) correspond à l'avenue de Chabeuil. Il semble bien, pense M. Bradford, qu'il ait été le decumanus principal non seulement de la ville mais aussi de son territoire, au moins du côté Est. Dans la ville, la disposition des rues modernes permet de reconnaitre, comme sur la photo, deux decumani secondaires au Sud du principal (rue Jonchères et rue du Général-Farre abou- 
tissant d̀ la place du Palais) et deux autres decumani secondaires au Nord (rue Belle-Image et rue Citadelle).

I.e croquis de M. Bradford que nous reproduisons (fig. 1), dressé d'après la photographie aérienne montre, à l'Est traces de la limitalio romaine se sontelles particulièrement conservées en cet endroit? C'est que les chemins établis dès lors répondaient à des besoins de circulation demeurés permanents alors qu'ailleurs la circulation locale s'est

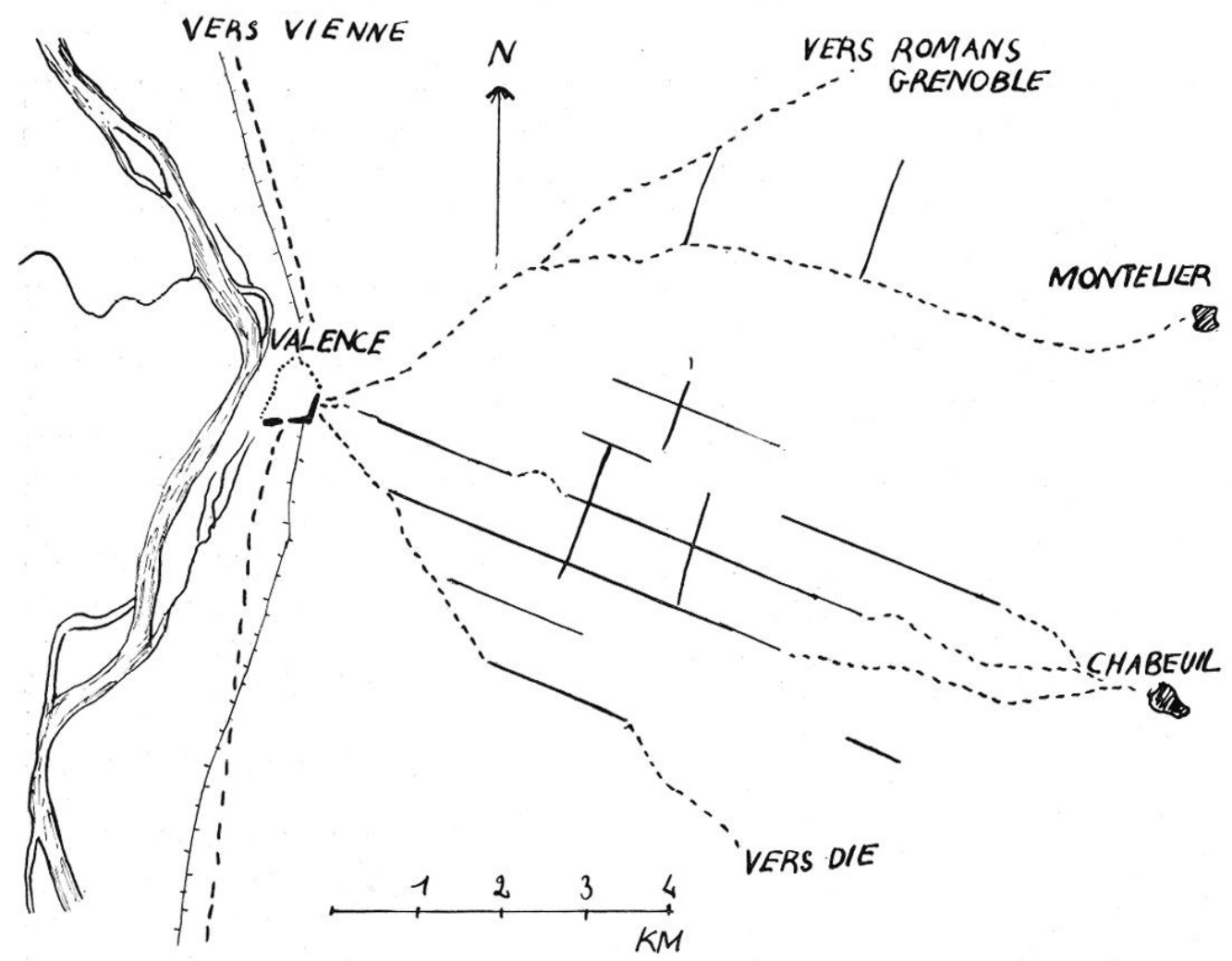

Fig. 1. - Valence (Drôme). Centuriation romaine.

D'après J. Bradford, Ancienl Landscapes, fig 20, p. 207.

de la ville: au moins, des tronçons de six decumani dont le principal est celui qui va vers Chabeuil. Le dessin des champs conserve, précise M. Bradford, la cenluriation romaine; il a stabilisé, dit-il, le plan de la colonie tel que l'ont établi les décemvirs qui l'ont installée. Cette régularité, remarque-t-il, distingue nettement cette partie de la plaine des régions situces au Nord ou au Sud, en remontant ou en descendant la vallée du Rhône. Pourquoi, se demande M. Bradford, les plus ou moins modifiée. M. Bradford ne manque pas de rappeler les indications donnécs naguère par M. A. Blanc sur les traces romaines conservées par le territoire à l'Est de Valence (Valence Romaine, 1953 et Revue des Éludes Ligures, 1953, p. 35-42) mais son croquis y ajoute des précisions et une certitude nouvelles.

Je ne puis insister sur toutes les observalions que l'analyse de la photographie aérienne suggère à $M$. Bradford sur le site même de Valence el sur les traces 


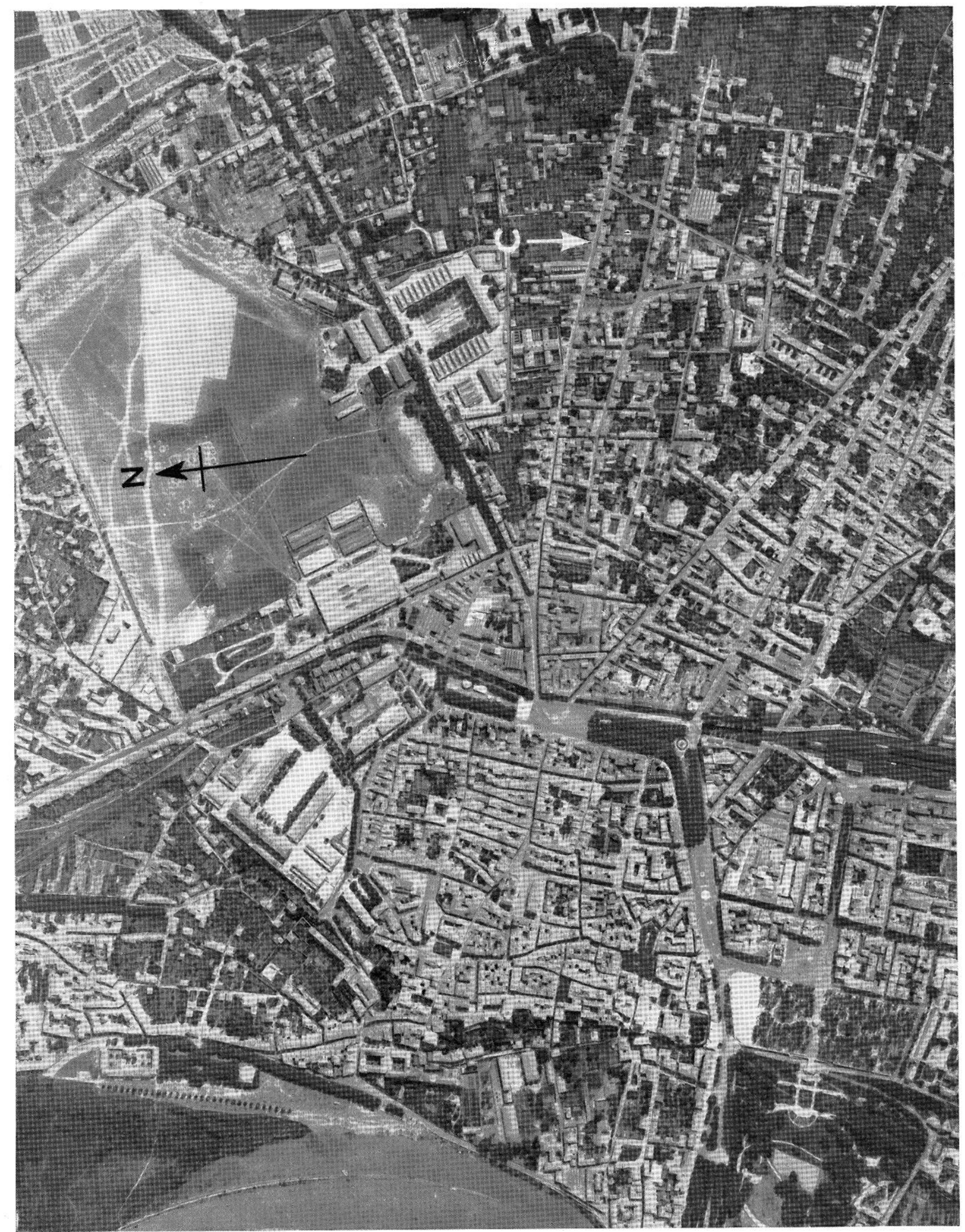

Fig. 2. - Le site de Valence. Photographie aérienne extraite de J. Bradford, Ancient Landscapes, aimablement communiquée par l'Auteur. 
laissées par les anciens bras latéraux du Rhône. Ces canaux sont aujourd'hui colmatés, mais la photographie permet de constater les traces laissées sur le terrain par les marécages qui les ont longtemps remplacés (fig. 2). Cependant je ne veux pas manquer de signaler le départ des grandes routes antiques qui convergent vers Valence. On les apercoil s'irradiant hors de la Porte de l'Est de la ville, de part et d'autre du decumanus de Chabeuil : celle de Vienne, vers le Nord ; vers le N.-E., celle de Romans et Grenoble et, au S.-E., celle de Die devant aboutir au Mont Genèvre et à Turin. Ce noeud de routes avait fait la prospérité de Valence, prospérité modeste, sans doute, d'une cité provinciale qui, en gardant le plan romain, a conservé le témoignage de deux mille ans de vie urbaine, tradilion qui a contribué efficacement à la civilisation générale de l'Europe méridionale et occidentale.

L'exemple de Valence, soulignc M. Bradford, apporte de précieuses indications en faveur de la recherche de la centuriation romaine autour d'autres colonies romaines de la Provence et, notamment, d'Orange. Les nombreux fragments d'inscriptions étudiés par le Chanoine Sautel el M. Piganiol dans Gallia (XIII, 1, 1955) témoignent de l'existence de cinq cadastres ruraux mais, jusqu'ici, on n'en a pas relevé les traces sur le terrain. A demie effacées cl moins claires qu'à Valence, ces traces existent cependant, dit M. Bradford (p. 211) et il se propose de les étudier. Promesse dont nous prenons acte et dont nous souhaitons voir l'heureuse réalisation ainsi que la publication définitive des cadastres ruraux de M. Piganiol.

\section{A. Grenier.}

\section{Deux inscriptions inÉdites de Vienne (ISÈre)}

Deux inscriptions, trouvćes l'une à Vienne et l'autre à proximité immédiate, sont venues enrichir le musée lapidaire installé dans l'ancienne église SaintPierre. Elles ont été inscrites sur le registre d'entrée sous les numéros 678 et 689. Le numéro 678 a été trouvé le 30 septembre 1955, à Vienne même, dans les travaux de fondation pour la construction d'un nouvel immeuble, rue Delorme, devant le porche de l'église Saint-Picrre. La picrre a été apportée au musée dans le courant du mois d'octobre 1955. Le numéro 689 a été trouvé dans le Rhône, en 1922, au lieudit SaintChrist, commune de Revenlin-Vaugris, par une personne qui l'a réemployé dans la construction de sa maison; encastrée d'abord dans le mur de cette maison, à la base de l'un des angles, la pierre a été transportée au musée le 17 avril 1956. Il s'agit de deux inscriptions funéraires, contribuant toutes deux à enrichir la prosopographie viennoise, au reste d'intérêt différent et inégal ${ }^{1}$.

1. La pierre no 689 est un bloc parallélépipédique en pierre de Choin (calcaire dur, propice à la gravure), en assez bon élal de conservation. L'inscription, disposée sur cinq lignes, est contenue dans

(1) Je remercie $M . \Lambda$ drien Bnuni, directeur des $\Lambda$ ntiquilés historiques de la circonscription de Grenoble, qui m'a libéralement accordé l'autorisation de publier ces deux inscriptions. Je tiens aussi à exprimer ma reconnaissance envers M.M. P.-M. Duval, J. Guey, H.-G. Prifaum et P. Wuilleumier pour leurs précieux conseils. 